\title{
Springer
}

\section{Blockchain-based approach to create a model of trust in open and ubiquitous higher education}

\author{
Journal of Computing in Higher Education \\ pp 1-26| Cite as
}

Article

First Online: 23 February 2019

\section{Abstract}

Currently, the training of the future work force presents challenging problems to higher education. This training, in the form of practical and theoretical knowledge can come from multiple platforms, channels and means, both formal and informal. In addition, it is quite difficult to assess the knowledge skill level that a student has acquired to optimize their chances for future employability. This, together with the need to still manage academic curricula on paper, the problems of confidence when validating these documents and contrasting them with real knowledge, etc., means that management in higher education requires revolutionary new tools. This work evaluates the benefits of the blockchain (or distributed ledger) technology and advocates a decentralised model of confidence for transactions based on an academic crypto currency. In this approach blockchain is used to manage transactions of content, teaching and competencies, assessed by consensus by students, trainers and employers, to eliminate once and for all the "gap" between the academic world and the working world. This paper aims to address the current challenges of an increasingly dispersed, open and ubiquitous higher education. The proposed model can be implemented in any training institution to adapt its teaching to the specific needs of professional profiles validated by employers in the sector. This model has been validated by means of a prototype with more than acceptable results.

\section{Keywords}

Blockchain Digital certificate Competencies Mined by consensus Confidence $\mathrm{P}_{2} \mathrm{P}$

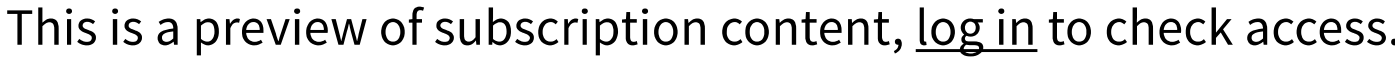




\section{Notes}

\section{Compliance with ethical standards}

\section{Conflict of interest}

The authors declare that they have no conflict of interest.

\section{Publisher's Note}

Springer Nature remains neutral with regard to jurisdictional claims in published maps and institutional affiliations.

\section{References}

Bartolomé, A. R., Bellver, C., Castañeda, L., \& Adell, J. (2017). Blockchain in education: Introduction and review of the state of the art. EDUTEC, Revista Electrónica de Tecnología Educativa (Electronic J ournal of Educational Technology), 61, 1-14. Google Scholar (http://scholar.google.com /scholar_lookup?title=Blockchain\%2oin\%2oeducation \%3A\%20Introduction\%20and\%2oreview\%20of\%20the\%20state\%20of\%2othe\%2 oart\&author=AR.\%20Bartolom\% 3 \%A9\&author $=$ C.\%20Bellver\& author=L.\%20Casta\%C3\%B1eda\&author=J.\%20Adell\&journal=EDUTEC \%2C\%2oRevista\%2oElectr\%C3\%B3nica\%20de\%20Tecnolog \% $3 \%$ ADa\%2oEducativa \%20\%28Electronic\%20Journal\%20of\%20Educational\%20Technology\%29\& volume $=61 \&$ pages $=1-14 \&$ publication_year $=2017$ )

Buterin, V. (2015). Understanding serenity, part 2: Casper. 28 December 2015. https://blog.ethereum.org/2015/12/28/understanding-serenity-part-2-casper/ (https://blog.ethereum.org/2015/12/28/understanding-serenity-part-2-casper/).

Cano, E., \& Cabrera, N. (2016). Competency assessment tool (CAT). The evaluation of an innovative competency-based assessment experience in higher education. J ournal Technology, Pedagogy and Education, 25(5), 631-648.

CrossRef (https://doi.org/10.1080/1475939X.2015.1134635)

Google Scholar (http://scholar.google.com /scholar_lookup?title=Competency\%20assessment\%2otool \%20\%28CAT\%29.\%20The\%2oevaluation\%20of\%20an\%2oinnovative\%2ocompete ncy-based\%20assessment\%2oexperience\%2oin\%2ohigher\%2oeducation\& author=E.\%20Cano\&author=N.\%20Cabrera\&journal=Journal\%20Technology $\% 2 \mathrm{C} \% 20$ Pedagogy $\% 20$ and $\% 20$ Education $\&$ volume $=25 \&$ issue $=5 \&$ pages $=631-648 \&$ publication_year=2016)

Clow, D., \& Makriyannis, E. (2011). iSpot analysed: Participatory learning and reputation. In: Proceedings of the 1st international conference on learning 
analytics and knowledge, 28 February- 01 March 2011, Banff, Alberta (pp.

34-43).

Google Scholar (https://scholar.google.com/scholar?q=Clow\%2C\%2oD.\%2C\%20

\%26\%20Makriyannis\%2C\%20E.\%20\%282011\%29.\%20iSpot\%2oanalysed

\%3A\%20Participatory\%2olearning\%2oand\%2oreputation.\%20In

\%3A\%20Proceedings\%20of\%20the\%201st\%2ointernational\%2oconference\%20on

\%2olearning\%20analytics\%20and\%2oknowledge\%2C\%2028\%20February

\%E2\%80\%9301\%20March\%202011\%2C\%2oBanff\%2C\%2OAlberta

\%20\%28pp.\%2034\%Е2\%80\%9343\%29.)

EdgeCoin Project [Online]. Available at: http://www.edgecoin.io/

(http://www.edgecoin.io/). Accessed in April 2018.

Jones, H. (2016). Broker ICAP says first to use blockchain for trading data.

Reuters, London, 15 March 2016. http://uk.reuters.com/article/us-icap-markets-

blockchain-idUKKCNoWH2J7 (http://uk.reuters.com/article/us-icap-markets-

blockchain-idUKKCNoWH2J7).

Knowledge Works, blockchaindailynews. (2016). [Online]. Available at:

https://www.blockchaindailynews.com/attachment/756565/

(https://www.blockchaindailynews.com/attachment/756565/). Accessed in April

2018.

Learningisearning, learningisearning2026 [Online]. Available at:

http://www.learningisearning2026.org/

(http://www.learningisearning2026.org/). Accessed in April 2018.

MIT, blockcerts, [Online]. Available at: https://www.blockcerts.org/

(https://www.blockcerts.org/). Accessed in April 2018.

Nakamoto, S. (2008). Bitcoin: A peer-to-peer electronic cash system. Available at: https://bitcoin.org/bitcoin.pdf (https://bitcoin.org/bitcoin.pdf). Accessed in April 2018.

Schlegel, H.: Reputation currencies. Institute of Customer Experience. http://ice.humanfactors.com/money.html (http://ice.humanfactors.com /money.html). Accessed in April 2018.

Sharples, M., \& Domingue, J. (2016), The Blockchain and Kudos: A distributed system for educational record, reputation and reward. In: Verbert K., Sharples M., Klobučar T. (eds) Adaptive and adaptable learning. EC-TEL 2016. Lecture notes in computer science (Vol. 9891). Springer.

Google Scholar (https://scholar.google.com/scholar?q=Sharples\%2C\%2oM.

\%2C\%20\%26\%2oDomingue\%2C\%20J.\%20\%282016\%29\%2C

\%20The\%2oBlockchain\%2oand\%20Kudos

\%3A\%20A\%2odistributed\%20system\%2ofor\%2oeducational\%2orecord \%2C\%2oreputation\%20and\%2oreward.\%20In\%3A\%2oVerbert\%2oK. \%2C\%20Sharples\%20M.\%2C\%2oKlobu\%C4\%8Dar\%20T.\%20\%28eds \%29\%20Adaptive\%20and\%20adaptable\%2olearning.\%2oEC-

TEL\%202016.\%20Lecture\%20notes\%20in\%20computer\%20science \%20\%28Vol.\%209891\%29.\%20Springer.)

Sony Global Education. Sony global education develops technology using Blockchain for open sharing of academic proficiency and progress records, 22 February 2016. http://www.sony.net/SonyInfo/News/Press/201602/16-0222E /index.html (http://www.sony.net/SonyInfo/News/Press/201602/16-0222E /index.html). Accessed in April 2018.

Tapscott, D., \& Tapscott, A. (2016). Blockchain revolution: How the technology behind Bitcoin is changing money, business, and the world. Brilliance Audio. 
Google Scholar (https://scholar.google.com/scholar?q=Tapscott\%2C\%2oD.

\%2C\%20\%26\%20Tapscott\%2C\%20A.

\%20\%282016\%29.\%20Blockchain\%2orevolution

\%3A\%20How\%20the\%2otechnology\%2obehind\%2oBitcoin\%2ois\%2ochanging\%2

omoney\%2C\%2obusiness

\%2C\%20and\%20the\%20world.\%2oBrilliance\%2oAudio.)

University of Nicosia. Academic Certificates on the Blockchain.

http://digitalcurrency.unic.ac.cy/free-introductory-mooc/academic-certificateson-the-blockchain/ (http://digitalcurrency.unic.ac.cy/free-introductorymooc/academic-certificates-on-the-blockchain/). Accessed in April 2018.

Valenzuela, J. (2016). Arcade City: Ethereum's Big Test Drive to Kill Uber. The Cointelegraph, March 15 2016. http://cointelegraph.com/news/arcade-cityethereums-big-test-drive-to-kill-uber (http://cointelegraph.com/news/arcadecity-ethereums-big-test-drive-to-kill-uber).

\section{Copyright information}

\section{(C) Springer Science+Business Media, LLC, part of Springer Nature 2019}

\section{About this article}

Cite this article as:

Lizcano, D., Lara, J.A., White, B. et al. J Comput High Educ (2019). https://doi.org/10.1007/s12528-019-09209-y

\section{First Online}

23 February 2019

\section{DOI}

https://doi.org/10.1007/s12528-019-09209-y

\section{Publisher Name}

Springer US

\section{Print ISSN}

1042-1726

\section{Online ISSN}

1867-1233

About this journal 
Reprints and Permissions

SPRINGER NATURE

(c) 2018 Springer Nature Switzerland AG. Part of Springer Nature. Not logged in · Not affiliated · 88.19.8.226 